NASA Technical Memorandum 83728

\title{
Characteristics of Arc Currents on a Negatively Biased Solar Cell Array in a Plasma
}

David B. Snyder

Lewis Research Center

Cleveland, Ohio

Prepared for the

Nuclear and Space Radiation Effects Conference sponsored by the Institute of Electrical and Electronics Engineers

1. Colorado Springs, Colorado, July 22-25, 1984

\section{N/SN}




\title{
CHARACTERISTICS OF ARC CURRENTS ON A NEGATIVELY BIASED SOLAR CELL
}

\section{ARRAY IN A PLASMA}

\author{
David B. Snyder \\ National-Aeronautics and Space Administration \\ Lewis Research Center \\ Cleveland, Ohio 44135
}

\begin{abstract}
SUMMARY
The time dependence of the emitted currents during arcing on solar cell arrays is being studied. The arcs are characterized using three parameters: the voltage change of the array during the arc (1.e., the charge lost), the peak current during the arc, and the time constant describing the arc current.

This paper reports the dependence of these characteristics on two array parameters, the interconnect bias voltage and the array capacitance to ground. It was found that the voltage change of the array during an arc is nearly equal to the bias voltage. The array capacitance, on the other hand, influences both the peak current and the decay time constant of the arc. Both of these characteristics increase with increasing capacitance.
\end{abstract}

\section{INTRODUCTION}

It is well known that if a shorted dark solar cell array is biased sufficiently negative in the presence of a plasma, it will exhibit arc discharges (refs. 1 to 4). While characteristic curves for simflar arcs have been pub1 ished (refs. 4 to 6 ), there has been no work suggesting parameters to characterize the arcs, or studying how conditions change the arcs. The purpose of this paper is to suggest parameters which describe the characteristics of the current from the array during these arcs. From past experience with solar array arcing it was decided to use three parameters to describe the arcs; the peak current of the arc, the decay time constant of the arc current, and the charge lost during the arc. This work also investigates the relationships between the arc characteristics and the array parameters of capacitance and bias voltage. This information will be useful in predicting the affects arcing will have on high power solar cell arrays in low Earth orbit.

Figure 1 shows the time dependence of the current from the array during a typical arc. These arcs are very similar to those reported due to interactions of electron beams of energies of several $\mathrm{keV}$ with solar cell arrays (ref. 5) and similar metal-insulator geometries (ref. 6). The arc has four time regions. First the arc is initiated and the current increases to a maximum; the rate of the increase varies considerably. Second, the current then stays near the peak value for some time. This current may be important in assessing damage to array materials, since resistive heating may cause some material to be bolled off the array. Third, the current decreases in a roughly exponential decay. This decay time is not a measure of the width of the arc. Finally, the arc terminates and the array begins to recharge to the bias voltage. The arc can be described by peak current, the time constant for the decay and the total charge lost during the arc. 
Two easily adjusted parameters in the experiment are the bias voltage applied to the interconnects, and the capacitance between the array and ground. The bias can be related to the operating voltage of a space system, particularly to the voltage difference between the arc point and the plasma. The capacitance may be an important parameter in scaling arcing effects to large area solar cell array power systems.

Both these parameters would be expected to influence the arc characteristics. The roughly exponential decrease in.current is suggestive of an RC time constant. In addition, both these parameters influence the amount of charge stored on the array, and thus, can be expected to change the characteristics of the arc.

\section{EXPERIMENT}

This experiment obtains arc characteristics from a biased solar cell array subjected to a plasma environment. A schematic illustration of the experiment. is shown in figure 2. A high voltage power supply is used to bias the array. A large resistance decouples the array from the power supply and its cable by limiting the charge able to flow to the array during an arc, yet it allows the array to be held at nearly the bias voltage. The capacitance of the array to ground is known, and additional capacitance can be introduced. The arcing characteristics are determined by using a capacitively coupled probe to measure the rate of change of the solar cell array voltage. The voltage changes can be related to the charge lost during an arc when the capacitance of the system is known.

The solar cell array (fig. 3) used here has been used in previous arcing studies (ref: 4). It is a 24 cell array segment, constructed of 2 crn square cells. The key feature, for the purposes of this experiment, is a copper plate on the fiberglass support of the array which has a capacitance of $65 \mathrm{pF}$ to the array. This is used as a capacitively coupled probe of the array voltage. The current to this back plate indicates the change in average voltage of the array. The 50 ohm resistance across the transient recorder gives a time con stant of $35 \mathrm{~ns}$, for this part of the circuit with the connecting cable; much shorter than the time scale of the arcs. This effectively holds the back plate at ground so the transient recorder reads the current to the back plate instead of the voltage of the back plate. This current multiplied by $65 \mathrm{pF}$ is the. change in voltage of the array.

Including additional capacitances in the system, enables this voltage change to be related to the net current from the array. The capacitance between the array and the supports, tank, etc. was found to be $50 \mathrm{pF}$. This capacitance was found by determining the charging time constant for the array through a known resistance. Capacitors having values of 235,1000 , and $2000 \mathrm{pF}$ can be added to begin to simulate the effect of larger area solar cell array-satellite systems in space. Knowing the capacitances involved in the experiment enables net current, i.e. the difference between the arc current and the return current to the cover slides, to be calculated.

The io Mohm carbon resistor decouples the array from the high voltage power supply. This resistance limits the array recharging time to greater than $1.15 \mathrm{~ms}$, much longer than the time scale of arcing. Additional capacitances increase this time. In addition, due to the not-quite-linear I-V character. istic of the resistor, the recharging current is imited to about $0.125 \mathrm{~mA}$, for 
a voltage drop across the resistor of $1000 \mathrm{~V}$. This current is smaller than the transient recorder can detect at the scale settings used for this work.

The arc characteristics were measured using two transient recorders. The data at capacitances of 115 and 350 pF were taken using a Biomation 610 transient recorder. This instrument has band widths of 2.5 and $2.0 \mathrm{MHz}$ at the scale settings used, sufficiently smaller than the decay time constants of the arcs investigated. During the higher capacitance tests, and for the $-500 \mathrm{~V}$ blas case a Blomation 6500 transient recorder was used. This instrument has a much higher band width, and also produced a considerably noisier signal. However, when smoothed numerically using a filtering time constant of about $0.1 \mu \mathrm{s}$, data from this instrument was consistent with data taken with the 610 . Care was taken to ensure that the arc signals were measured with an instrument of appropriate band width.

Three arc characteristic parameters were calculated using the digitally recorded history of each arc. The total charge loss was recorded as a change in voltage of the array during an arc. This was calculated by integrating the positive measured current (the recorded negative currents were ignored) over the time of the arc and multiplying by the $65 \mathrm{pF}$ capacitance between the back plate and the array. This gives a lower bound on the charge loss since the negative pulse at the arc's termination indicates that there is a small return current to the cover slides of the solar cells during the arc.

The peak current of the arc (Ip) was found by simply searching the arc's data set for the maximum value of the positive current. The decay time ( $t$ ) of the arc current was calculated using several steps. First, the natural logarithm of the arc current was plotted versus time and a region of the decay where this plot was nearly linear was determined. Next, the slope in that region was determined through a linear least squares fit. The decay time reported is the inverse of that slope.

Plasma parameters were obtained throughout the tests. A $1200 \mathrm{~cm}^{2} \mathrm{disk}$ was driven with a Tektronix 7CTIN Curve Tracer to examine the electron population characteristics. Typically the electron temperature was 1 to $2 \mathrm{eV}$, the plasma potential was +7 to $+10 \mathrm{~V}$, and the electron density was $10^{3}$ to $10^{4} \mathrm{~cm}^{-3}$. There was considerable varlation in these plasma parameters throughout a test run, apparently related to the applied bias voltage on the array. Attempts to correlate plasma parameters with arc characteristics were unsuccessful for this range of plasma parameters.

\section{RESULTS AND DISCUSSION}

Charge loss and Interconnect Blas Voltage. - The charge lost during an arc was measured for interconnect bias voltages between -500 and $-1000 \mathrm{~V}$. A summary of these measurements is shown in figure 4. It is convenient to express the charge loss during an arc as a change in voltage since this provides an easy comparison with the blas voltage. The charge loss and the change in voltage are related by the capacitance of the system.

The relationship between the change in voltage and interconnect bias voltage is shown in figure 4 (a) and is straightforward. The two are nearly equal. During a solar array arc, from half to nearly all the charge stored between the solar array and ground is lost. Typlcally, 75 to 80 percent of the charge is 
1ost. This caused the interconnect potential to go to about $200 \mathrm{~V}$ from plasma ground. This has an important implication in that during an arc on a space power system, the arc point would be brought toward plasma ground.

Figure 4 (b) shows the result of changing the capacitance of the system. There is no obvious relationship between the capacitance and the voltage change of the system during an arc. This indicates that the voltage change of the system is a more useful parameter describing the arcs space systems than the charge loss, since the voltage change includes the capacitance information and is readily compared to the interconnect voltage at a specified arc point.

Peak Arc Current and Arc Decay Time. - Figure 5 shows peak currents, Ip, and decay times, $t$, under three conditions. These data sets fllustrate the changes in probable value for Ip and $t$ of an arc, caused by changing bias voltage and by changing capacitance. From the $350 \mathrm{pF}$ data, it appears that Ip and $t$ are related to each other.

While a linear least squares fit to each data set is possible, a more natural procedure is to examine the product Ipt. This quantity has the units of charge, and would indicate the voltage dron of the array during the decay part of the arc if the arc did not terminate. It may represent the array voltage at which the current starts to decay.

The curves drawn on the figure represent the average value of Ipt for each of the data sets. It is seen that decreasing the bias voltage decreases Ipt only slightly, if at all. As the capacitance of the system is decreased, Ipt is decreased, which is consistent with the idea that the arc current decay can be modeled as an RC decay, 1.e., as the capacitance decreases the time constant of the decay decreases. Apparently the bias voltage effects primarily the early part of the arc and not the decay region.

Even though a relationship between $I_{p}$ and $t$ would be expected since the total change in voltage is roughly the same for each of these arcs, this is not the reason for the correlation. Figure 6 shows a plot of Ipt versus change in voltage for a set of arcs. This scatter plot shows that the two data sets are unrelated. This figure demonstrates that in an arc's current history, the total charge lost is unrelated to the size of the arc decay region. The region of rising current and the region of peak current contain a major contribution to the total charge lost.

Ipt and Capacitance. The notion that capacitance of the array system plays an important role in the determination of arc characteristics is intriguing. It is of particular interest that the arc current should be influenced by the capacitance. This is investigated further in figure 7 , where the behavior of $I_{p}$ and $t$ is shown up to. $2115 \mathrm{pF}$.

Several observations are made. First, the scatter of the data increases with capacitance. This means that the conclusions made should be shown an appropriate amount of skepticism. Secondly, the average value of Ipt increases linearly with capacitance. This suggests again that the voltages involved, are more characteristic of the arcs than the amount of charge transferred. Next, the decay time constants appear to be leveling off as the capacitance increases. A log-log plot of average $t$ versus capacitance shows the same behavior. This suggests that the decay time may approach a limiting 
value. Finally, Ip appears to increase with capacitance. Since Ipt increases linearly with capacitance and if $t$ approaches a constant value, then Ip would increase at a rate nearly linear with capacitance. This suggests that in space, the more area a solar cell array/satellite has, the larger the currents drawn during an arc.

\section{CONCLUSION}

In this paper, three characteristic properties of solar cell array arcs were used to determine the effect of two design parameters on these arcs. The characteristics chosen are the peak current during an arc, the decay time of the current as the arc subsides, and the voltage drop of the solar cell array during the arc. The design parameters investigated are the interconnect blas voltage, related to the voltage difference between the plasma and the arc point on a space power system, and the capacitance of the array, related to the area of a high power solar cell array.

The following conclusions are drawn from the observations reported here:

(1) The change in voltage of the array during an arc ranged from half to nearly all the interconnect bias voltage, with a corresponding fraction of the stored charge being lost. Typically, the arc ended with the array about $200 \mathrm{~V}$ from ground. This suggests that when a solar cell array in a high power space system arcs, arc point will approach plasma ground.

(2) The product of the peak current and the arc decay time (Ipt) is linearly related to the capacitance of the array system. This may be relevant to the problem of scaling arc phenomenon from small arrays to very large arrays.

(3) The observed arc current decay times increase with increasing capacitance of the array, for small capacitances. However, at capacitances above $2000 \mathrm{pF}$ the decay time may be limited to a value of about $5 \mu \mathrm{s}$. The peak current increases with increasing capacitance, and since Ipt appears to increase linearly, may increase linearly with increasing capacitance for large capacitances. At $2115 \mathrm{pF}$ the peak currents were in the range of 0.3 to $0.7 \mathrm{~A}$.

The arc characteristic parameters used in this work allow comparisons between solar cell array operating parameters to be made. The data presented may allow some hypotheses to be formulated regarding the mechanism initiating solar array arcing in a plasma. It is expected that the experiment described here can produce additional information useful in modeling the effects of arcing on space power systems.

\section{REFERENCES}

1. Stevens, N. J., "Review of Blased Solar Array-Plasma Interaction Studies," NASA TM-82693, 1981.

2. Grier, N. T., and Stevens, N. J., "Plasma Interaction Experiment (PIX) Flight Results," in Spacecraft Charging Technology - 1978, AFGL-TR-790082, 1979, pp. 295-314. 
3. Grier, N. T., "Plasma Interaction Experiment II (PIXII): "Laboratory and Flight Results," to be published in Proc. Spacecraft Environmental Interactions Conference 1983, cosponsored by the U.S. Air Force and NASA.

4. Snyder, D. B., "Discharges on a Negatively Biased Solar Array in a Charged Particle Environment," to be published in Proc. Spacecraft Environmental Interactions Conference 1983, cosponsored by the U.S. Air Force and NASA.

5. Snyder, D. B., "Environmentally Induced Discharges in a Solar Array," IEEE Trans. Nucl. Sc1., vol. NS-29, 1982, pp. 1607-1609.

6. Leung, P., "Discharge Characteristics of a Simulated Solar Ce11 Array," IEEE Trans. Nuc 1. Sc1., vol. NS-30, 1983, pp. 4311-4315. 


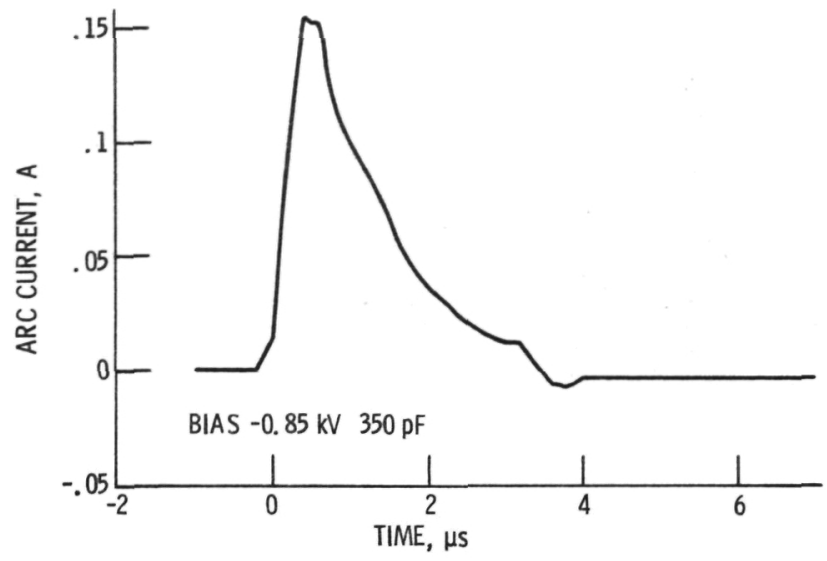

Figure 1. - Back plate signal during discharge.

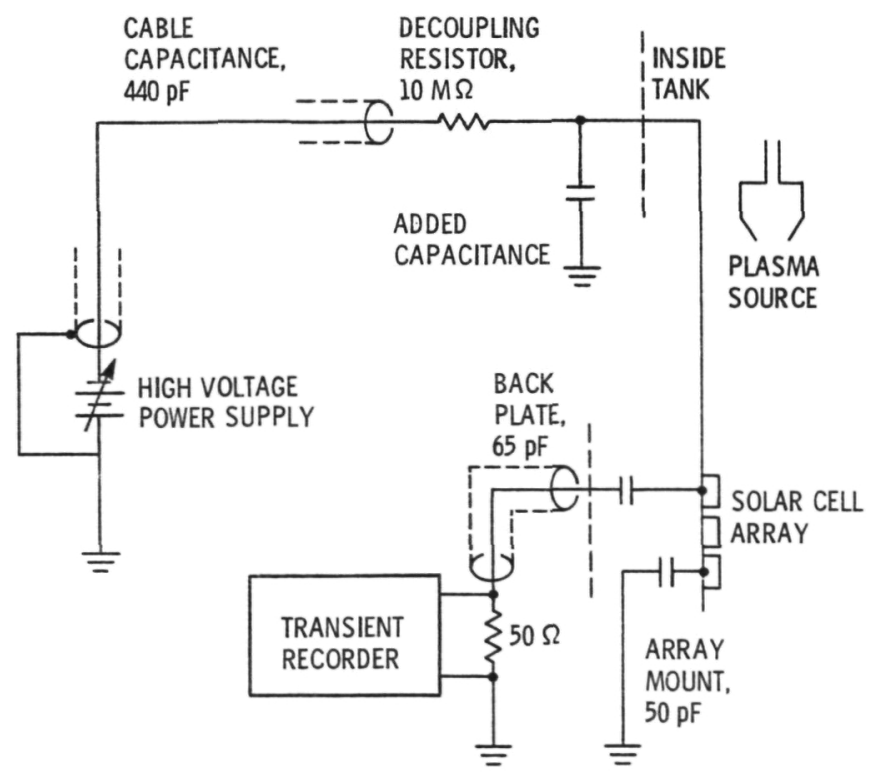

Figure 2. - Experiment schematic. 


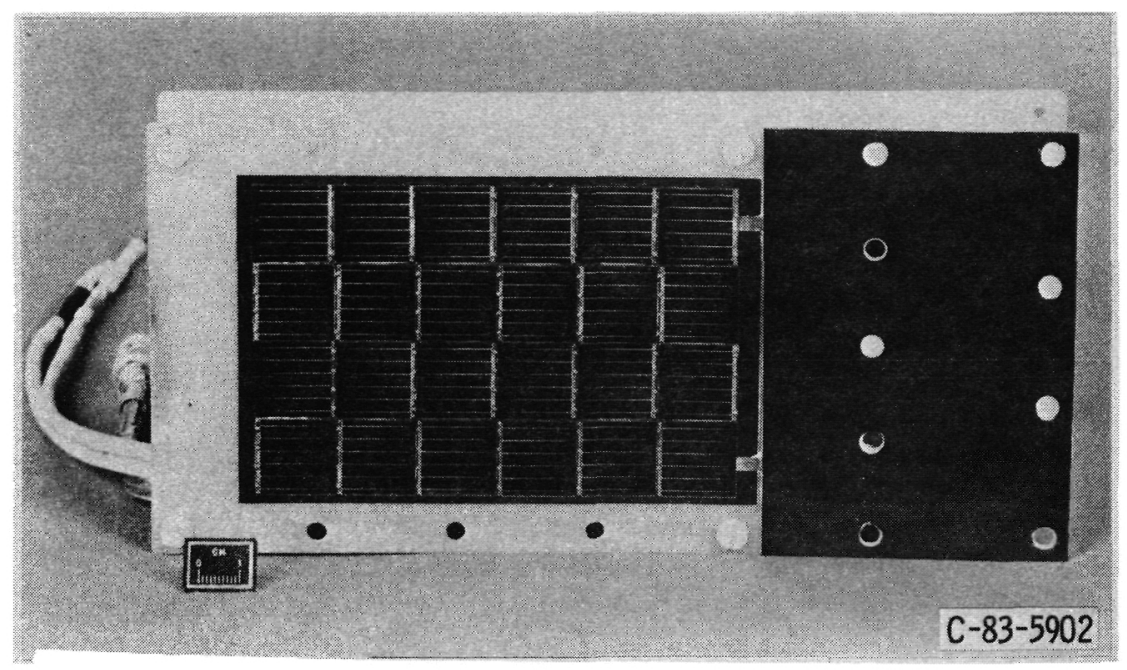

Figure 3。 - Solar array tested.

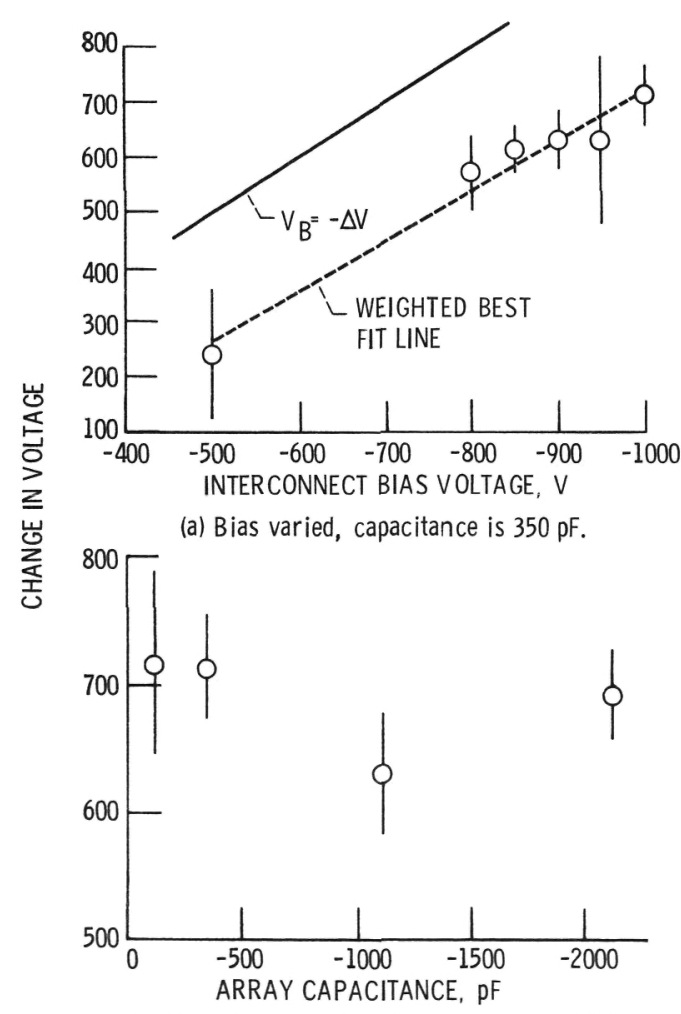

(b) Capacitance varied, bias voltage is $-1000 \mathrm{~V}$.

Figure 4. - Influence of bias voltage and array capacitance on the charge lost (change in voltage) during an arc. 


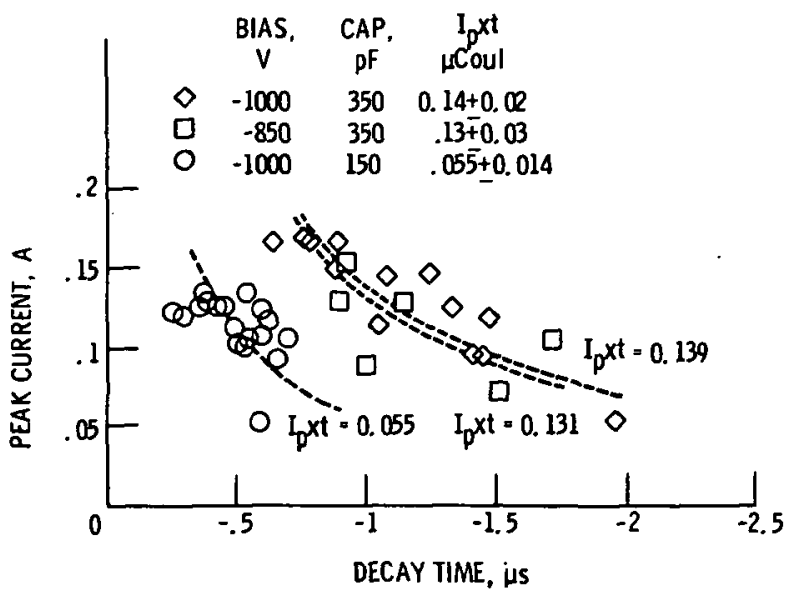

Figure 5. - The relation between peak current $\left(I_{p}\right)$ and decay time (t) during arcs.

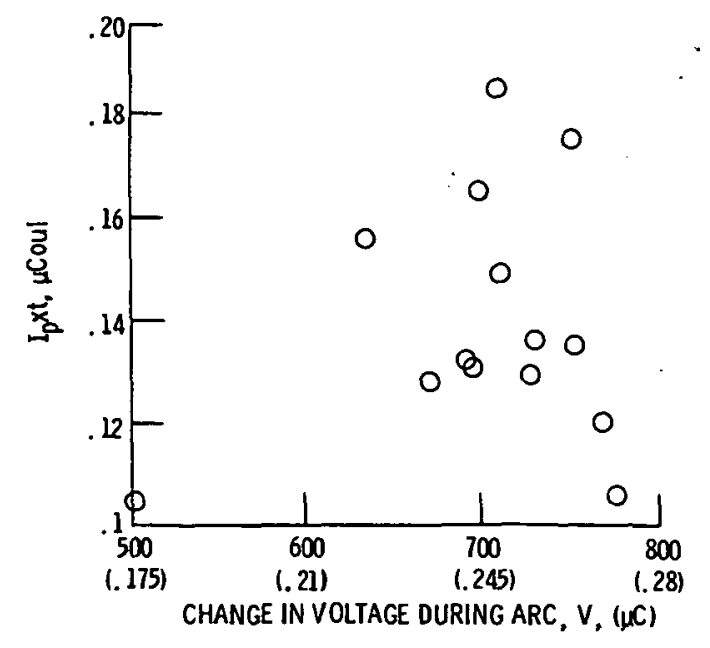

Figure 6. - Charge lost during decay compared with charge lost (voltage change), $-1000 \mathrm{~V}$ bias, $350 \mathrm{pF}$. 


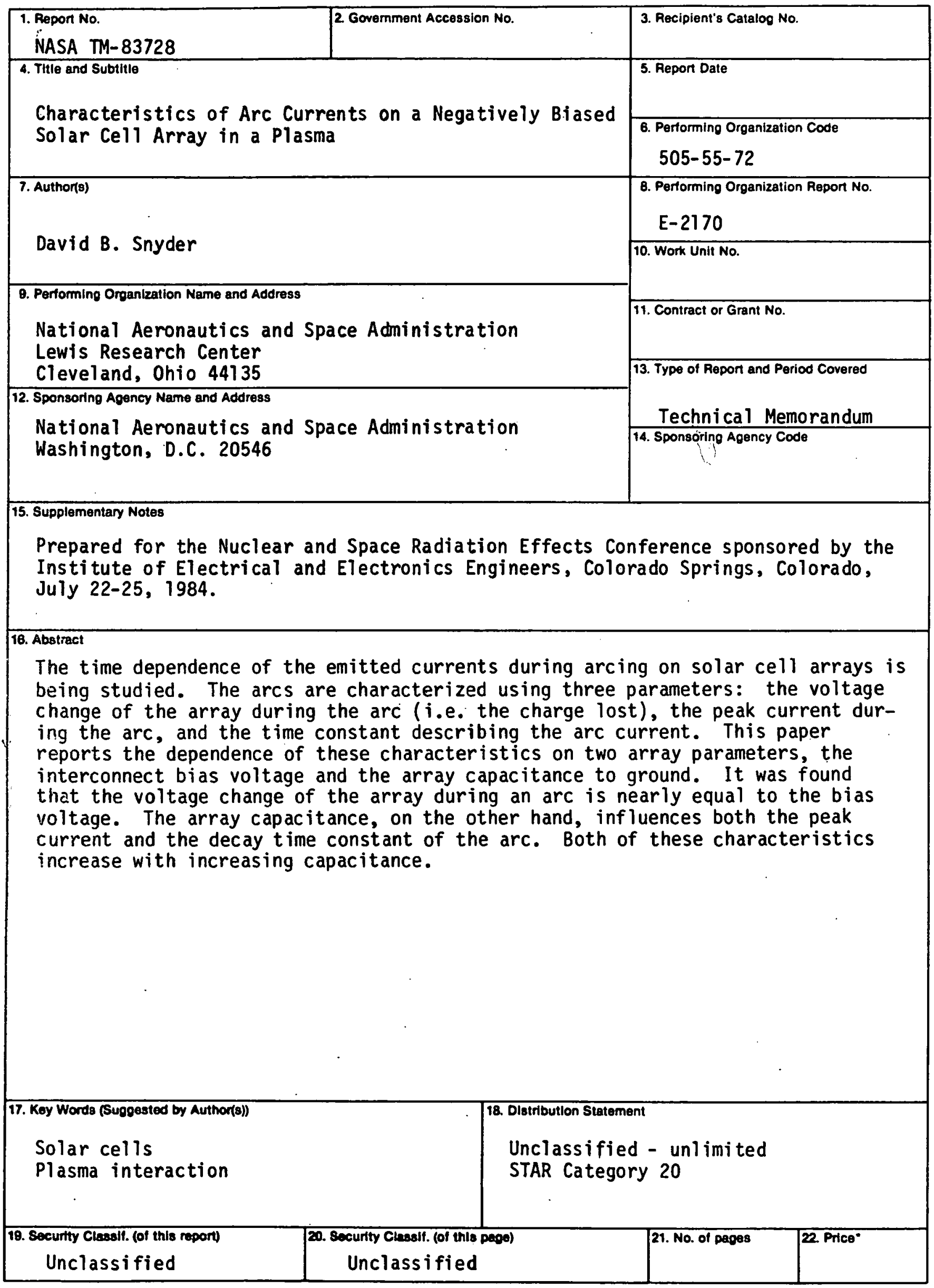

-For sale by the National Technical Information Service, Springfield. Virginia 22161 
National Aeronautics and Space Administration

Washington, D.C.

20546

Orficial Business

Penalty for Private Use. $\mathbf{5 3 0 0}$
SPECIAL FOUATH CLASS MAIL BOOK

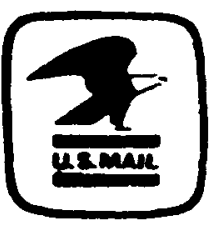

Portege and Fees Paid National Aeronoutics and Spece Administration NASA-451 